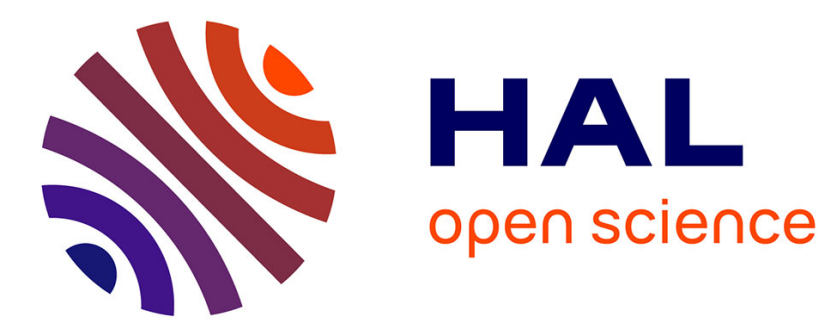

\title{
Pêches artisanales dans les petits barrages
}

Luis Tito de Morais, Kouassi Sebastino da Costa, Jean Raffray, Kassoum

Traoré

\section{To cite this version:}

Luis Tito de Morais, Kouassi Sebastino da Costa, Jean Raffray, Kassoum Traoré. Pêches artisanales dans les petits barrages. L'eau en partage : les petits barrages de Côte d'Ivoire, IRD, Paris, pp.215-227, 2007. hal-00495448

\section{HAL Id: hal-00495448 \\ https://hal.science/hal-00495448}

Submitted on 12 Sep 2010

HAL is a multi-disciplinary open access archive for the deposit and dissemination of scientific research documents, whether they are published or not. The documents may come from teaching and research institutions in France or abroad, or from public or private research centers.
L'archive ouverte pluridisciplinaire HAL, est destinée au dépôt et à la diffusion de documents scientifiques de niveau recherche, publiés ou non, émanant des établissements d'enseignement et de recherche français ou étrangers, des laboratoires publics ou privés. 
Luis Tito De Morais

Sebastino Da Costa

Jean Raffray

Kassoum Traoré

\section{Pêches artisanales}

dans les petits barrages

Les travaux portent sur une étude de suivi par enquêtes des pêches artisanales réalisée par l'Idessa en 1994 sur 4 petits barrages du nord de la Côte d'Ivoire, puis sur son extension à 9 retenues entre 1996 et 1998. Des pêches expérimentales (filets maillants et senne) ont alors été parallèlement réalisées sur 5 d'entre elles.

Ces diverses sources d'information sont utilisées ici pour, d'une part, évaluer les captures et leur valeur économique, et, d'autre part, décrire les modalités de l'exploitation halieutique en vigueur dans les petits barrages et commenter leurs possibilités d'amélioration.

L'étude de 1994 a été réalisée sur deux petites $\left(0,06\right.$ et $\left.0,085 \mathrm{~km}^{2}\right)$ et deux moyennes $\left(0,21\right.$ et $\left.0,44 \mathrm{~km}^{2}\right)$ retenues (IDESSA, 1992 ; DA COSTA et al., 1998). Les enquêtes réalisées de 1996 à 1998 ont concerné 9 retenues, présentant des tailles variant entre 0,08 et $0,45 \mathrm{~km}^{2}$ (fig. 1 et annexe). Sur 5 d'entre elles, des pêches expérimentales (filets maillants et senne) ont été pratiquées afin de comparer les captures artisanales du site aux peuplements de poissons échantillonnés sur place. Toutes ces retenues agropastorales, édifiées dans les années 1970 et 1980 sous l'égide de sociétés d'État, sont permanentes et situées sur des affluents du fleuve Bandama, sauf Nambengué, qui barre un affluent du Comoé. Pour chacune d'elles, la surface maximale théorique (IDESSA, 1992) ou mesurée (CoRBIN et ÉtIENnE, 1998) et la profondeur maximale mesurée (CoRbin et ÉtIENNE, 1998 ; YTÉ et al., 1997) ont été considérées. En année normale, les marnages observés sont de l'ordre de 1,5 m.

\section{Introduction}

\section{Matériel et méthodes}



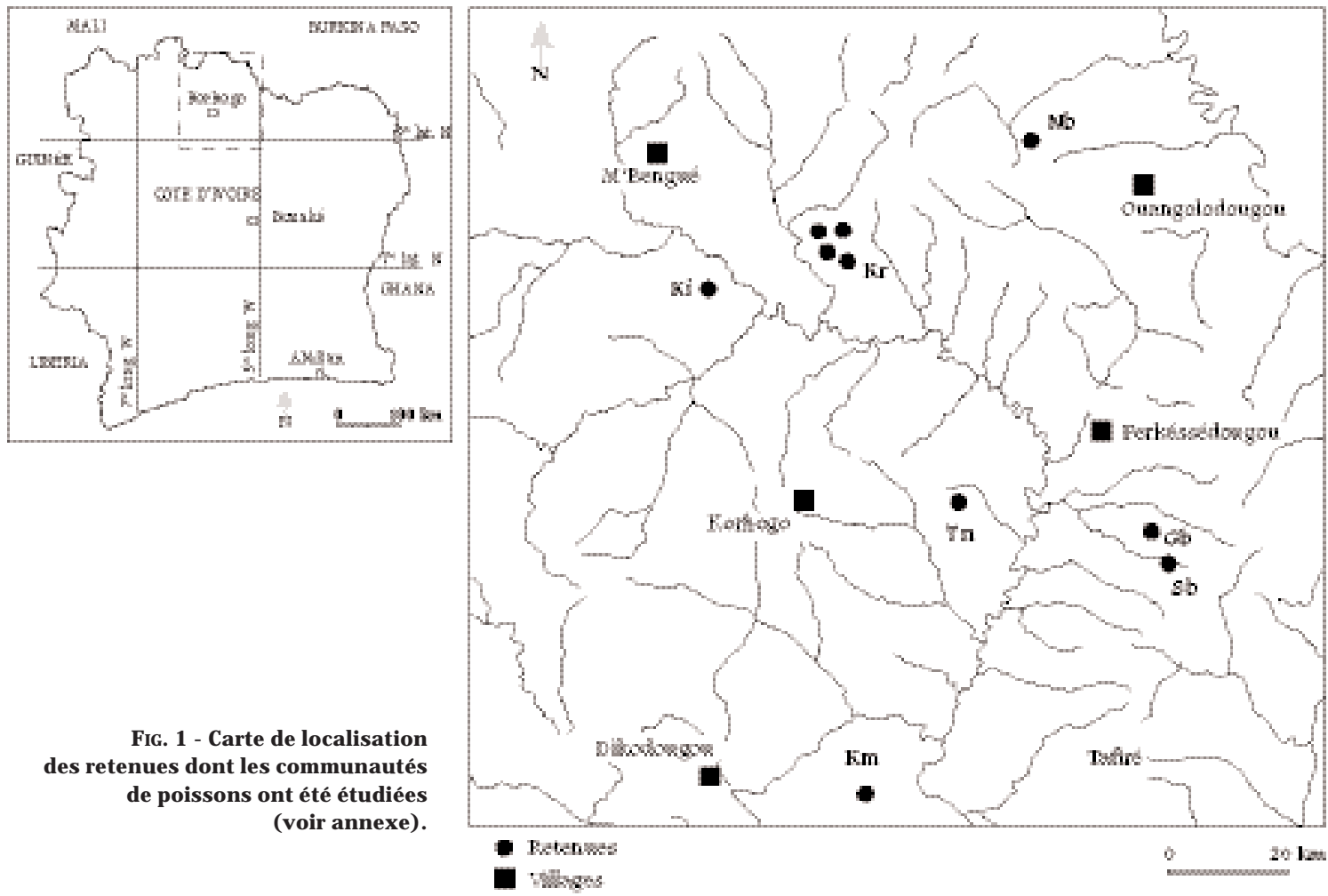

FIG. 1 - Carte de localisation des retenues dont les communautés de poissons ont été étudiées (voir annexe).

Whilkges

Les barrages étudiés sont : Kiémou, dit Kiémou Aménagé (Km), Sambakaha (Sb), Gboyo (Gb), Korokara Serpent, Korokara Termitière, Korokara Sud, Korokara Nord (tous les sites de Korokara sont repérés sur la figure 1 par le code $\mathrm{Kr}$ ), Tiné ( $\mathrm{Tn})$, Katiali (Ki), Nambengué ( $\mathrm{Nb}$ ). Les quatre premières retenues ont été étudiées en 1994 . Seul le premier site n'a pas fait partie du panel de l'étude réalisée entre 1996 et 1998. Les noms soulignés indiquent les réservoirs ayant fait l'objet de pêches expérimentales.

Des empoissonnements avec Oreochromis niloticus ont été réalisés par la Sodepra en 1984 et 1985 dans les retenues de Kiémou et Korokara Serpent, puis par l'Idessa et I'Orstom en mai 1997 dans les retenues de Nambengué, Katiali et Korokara Termitière. D'autres empoissonnements sur lesquels nous n'avons pas obtenu de données ont été réalisés en utilisant 0 . niloticus et Heterotis niloticus : ces deux espèces sont présentes dans toutes les retenues étudiées ici (Duponchelle et al., 2000). Lates niloticus a été introduit dans la retenue de Tiné (Kouassi et al., ce volume). Des Chrysichthys ont également été introduits par les pêcheurs à Korokara Serpent, sans succès durable semble-t-il. 
Les statistiques de pêche proviennent d'enquêtes réalisées «bordbarrage » au moment de la relève des filets et de la vente des poissons (en général, très tôt le matin). Les poissons ont été pesés par espèce à l'aide d'une balance mécanique d'une portée de $20 \mathrm{~kg}$. Les données répertoriées concernent les types d'engins et le nombre d'engins de chaque type utilisés, le temps de pêche, l'utilisation éventuelle d'intrants (appâts), leur nature, quantité et valeur, les espèces composant la prise, le nombre d'individus et le poids de la prise par espèce. Ces relevés ont été, sauf exception, quotidiens sur chaque barrage. L'unité d'effort retenue ici a été le « pêcheur jour-1 ».

À partir de ces enquêtes, les indices suivants ont été établis pour chaque retenue :

- nombre moyen de pêcheurs : pêcheurs jour-1 mois-1 ;

- production exploitée : $k g$ an-1;

- production exploitée par unité de surface : $\mathrm{km}^{-2} \mathrm{an}^{-1}$.

La nasse papolo est un engin de pêche très utilisé dans les petits barrages.

Déposées par les pêcheurs dans les zones peu profondes, les nasses sont parfois appâtées avec des boulettes de farine de maïs.

Trois ouvertures latérales permettent au poisson de pénétrer dans le piège. Cichlidés et siluriformes constituent la majeure partie des prises réalisées avec ce type d'engin.

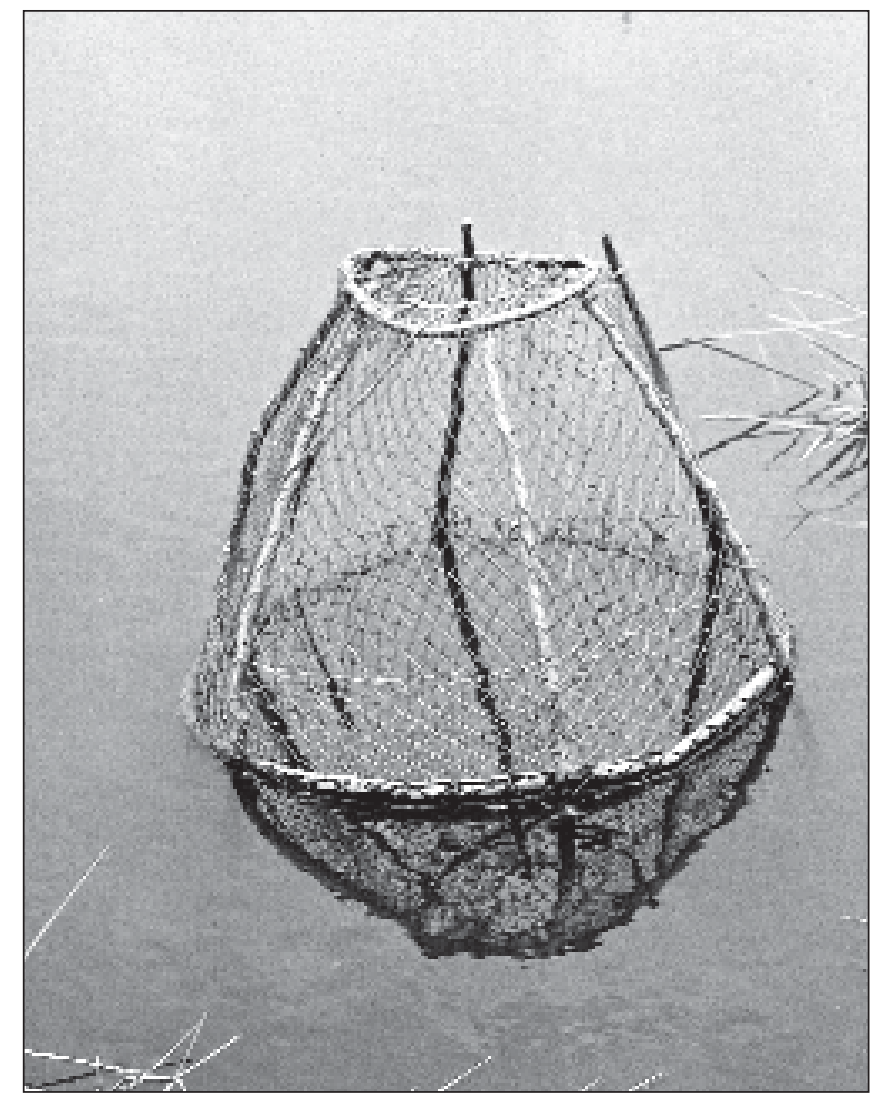


Nous avons effectué des comparaisons entre les productions exploitées des retenues étudiées et celles d'autres barrages, et recherché une éventuelle corrélation entre la production exploitée et la surface des retenues (modèle linéaire). Les compositions et les rangs des espèces dans les captures des pêches expérimentales et artisanales ont été comparés dans le but de mettre en évidence d'éventuels taxons non exploités dans le peuplement des retenues.

Sur la base d'une fourchette de prix de vente comprise entre une valeur minimale de $250 \mathrm{FCFA} \mathrm{kg}^{-1}$ (prix de vente bord-barrage des individus de petite taille) et un prix maximal de $500 \mathrm{FCFA} \mathrm{kg}^{-1}$ (prix de vente bord-barrage le plus couramment observé pour les tilapias de grande taille), les chiffres d'affaire ont été estimés directement, en appliquant ces prix aux quantités annuelles débarquées observées durant nos enquêtes.

L'efficacité moyenne des pêcheurs est celle observée en moyenne pour chaque retenue lors de l'ensemble de nos enquêtes; elle est exprimée en « kg pêcheur-1 jour-1 mois $^{-1}$ ».

La production totale annuelle exploitée par unité de surface pour chaque retenue est calculée et comparée aux valeurs de production exploitée indiquées dans la littérature et aux estimations empiriques de production pour les réservoirs de surface inférieure à $10 \mathrm{~km}^{2}$ (CRUL, 1992 b).

Résultats Du point de vue de l'effort de pêche, les retenues se répartissent en trois groupes (tabl. 1):

(1) trois retenues où l'effort de pêche est très faible, inférieur à 4 à 9 pêcheurs jour-1 mois $^{-1}$;

(2) quatre retenues où l'effort de pêche se situe entre 15 et 20 pêcheurs jour-1 mois $^{-1}$;

(3) trois retenues où l'effort de pêche est très élevé, toujours supérieur à 25 pêcheurs jour-1 mois $^{-1}$.

Les retenues à faible effort de pêche sont de très petits réservoirs (0,01 à $0,08 \mathrm{~km}^{2}$ ) proches du village de Korokara dont dépend aussi le barrage de Korokara Serpent qui est lui bien plus grand $\left(0,44 \mathrm{~km}^{2}\right)$ et fortement exploité (de 26 à 31 pêcheurs jour-1 mois $^{-1}$ en moyenne). Quatre pêcheurs ont opéré sur les trois retenues peu exploitées de Korokara, 


\begin{tabular}{|c|c|c|c|c|c|c|}
\hline Source & Retenue & $\begin{array}{c}\text { Surface } \\
\left(\mathrm{km}^{2}\right)\end{array}$ & $\begin{array}{c}\text { Effort de pêche } \\
\text { (pêcheurs jour-1 } \text { mois }^{-1} \text { ) }\end{array}$ & $\begin{array}{c}\text { P. exploitée } \\
\left(\mathrm{kg} \mathrm{jour}^{-1} \text { pêcheur }{ }^{-1}\right)\end{array}$ & $\begin{array}{c}\text { P. exploitée } \\
\left(\mathrm{kg} \mathrm{an}^{-1}\right)\end{array}$ & $\begin{array}{l}\text { P. exploitée } \\
\left(\mathrm{t} \mathrm{km}^{-2} \mathrm{an}^{-1}\right)\end{array}$ \\
\hline 2 & Korokara Nord & 0,01 & 7 & 3,3 & 55 & 6,7 \\
\hline 2 & Korokara Sud & 0,02 & 4 & 2,7 & 31 & 1,5 \\
\hline 2 & Koroakara Termitière & 0,08 & 9 & 5 & 265 & 3,5 \\
\hline 1 & Kiémou & 0,06 & 19 & 5,9 & 1333 & 22,4 \\
\hline 1 & Gboyo & 0,09 & 16 & 6,2 & 1183 & 13,9 \\
\hline 2 & Gboyo & 0,09 & 18 & 21,8 & 3626 & 42,6 \\
\hline 2 & Nambengué & 0,13 & 20 & 7,6 & 1527 & 11,6 \\
\hline 1 & Sambakaha & 0,21 & 20 & 14,9 & 3555 & 16,8 \\
\hline 2 & Sambakaha & 0,21 & 17 & 39,2 & 6814 & 32,2 \\
\hline 2 & Katiali & 0,31 & 35 & 9,2 & 3200 & 10,5 \\
\hline 1 & Korokara Serpent & 0,44 & 31 & 2,8 & 1062 & 2,4 \\
\hline 2 & Korokara Serpent & 0,44 & 26 & 6,6 & 1637 & 3,7 \\
\hline 2 & Tiné & 0,45 & 44 & 10,1 & 4905 & 10,9 \\
\hline 3 & Boulbé & 1,7 & - & - & - & 3,5 \\
\hline 3 & Petit Balé & 2,5 & - & - & - & 8 \\
\hline 3 & Nagbangré & 3 & - & - & - & 5 \\
\hline 3 & Sourou & - & - & - & - & 9,4 \\
\hline 3 & Mogtedo & 4,5 & - & - & - & 10 \\
\hline 3 & Tapoa & 8,5 & - & - & - & 20 \\
\hline 4 & Bahut & 2,1 & - & - & 180000 & 87,3 \\
\hline 4 & Pagusi & 2,5 & - & - & 25000 & 10 \\
\hline 4 & Dapao & 2,6 & - & - & 120000 & 46,1 \\
\hline 4 & Dapao & 4,8 & - & - & 500000 & 104,2 \\
\hline 4 & Yodaw ewa & 4,9 & - & - & 77000 & 15,7 \\
\hline 4 & Weerawila & 5,7 & - & - & 46000 & 8,1 \\
\hline
\end{tabular}

Tabl. I - Données de base sur la pêche dans les retenues étudiées et données de la littérature.

Surface : surface de la retenue.

P. exploitée (production exploitée) : quantité de poissons (poids) débarquée par les pêcheurs artisans.

mais il semblerait que ces sites ne constituent qu'une ressource marginale pour les pêcheurs de ce village, qui exploitent essentiellement la grande retenue de Korokara Serpent où 10 à 15 pêcheurs opèrent régulièrement simultanément.

Le groupe des retenues sur lesquelles s'observe un effort de pêche intermédiaire est plus hétérogène quant au nombre de pêcheurs qui l'exploitent.

Effort de pêche : moyenne mensuelle du nombre de pêcheurs observé chaque jour sur chaque retenue.

Sources :

1- DA Costa et al., 1998 :

2- Données de notre étude

3- Baljot et al., 1994 (Burkina Faso) ;

4- Moreau et De SILVA, 1991 (Philippines et Sri Lanka)]. 
Il y a dans certains cas un seul (ou deux) pêcheur(s) attitré(s) : Gboyo, Kiémou et Sambakaha en 1994. Durant nos enquêtes, de 7 à 9 pêcheurs se sont succédé sur les retenues de Sambakaha et Nambengué.

Le groupe de retenues où l'effort de pêche est le plus important correspond aux barrages de plus grandes tailles : Katiali $\left(0,31 \mathrm{~km}^{2}\right)$, Korokara Serpent $\left(0,44 \mathrm{~km}^{2}\right)$, et Tiné $\left(0,45 \mathrm{~km}^{2}\right)$.

Les quantités de poisson pêchées par unité de surface sont dans les mêmes ordres de grandeur que celles observées ailleurs en milieu tropical mais pour des retenues 10 fois plus grandes en moyenne (tabl. I). La production exploitée dans l'ensemble des retenues dépendant du village de Korokara est toujours très faible. Les retenues du groupe où l'effort de pêche est intermédiaire présentent le plus fort rendement par unité de surface (tabl. I). À l'exception des retenues de Gboyo et Sambakaha, les rendements actuels, comme ceux mentionnés par DA COSTA et al. (1998), s'adaptent bien au modèle de CRUL (1992 b) (ronds noirs et gris respectivement, fig. 2 ).

Sous l'hypothèse d'une standardisation de l'effort de pêche à 30 pêcheurs jour ${ }^{-1}$ mois $^{-1}$, les rendements prédits restent également bien adaptés au modèle (ronds blancs sur la fig. 2). Les retenues de Gboyo et Sambakaha présentent des points excentrés en raison de l'efficacité apparente des pêcheurs dont le rendement journalier moyen est largement supérieur à celui des autres (tabl. I). La retenue de Korokara Serpent affiche inversement des rendements très faibles.

À l'exception de Schilbe intermedius, peu présent dans les pêches artisanales, les espèces les plus importantes (en poids) dans les peuplements décrits par les pêches expérimentales sont également celles qui se retrouvent dans les pêches artisanales (tabl. II).

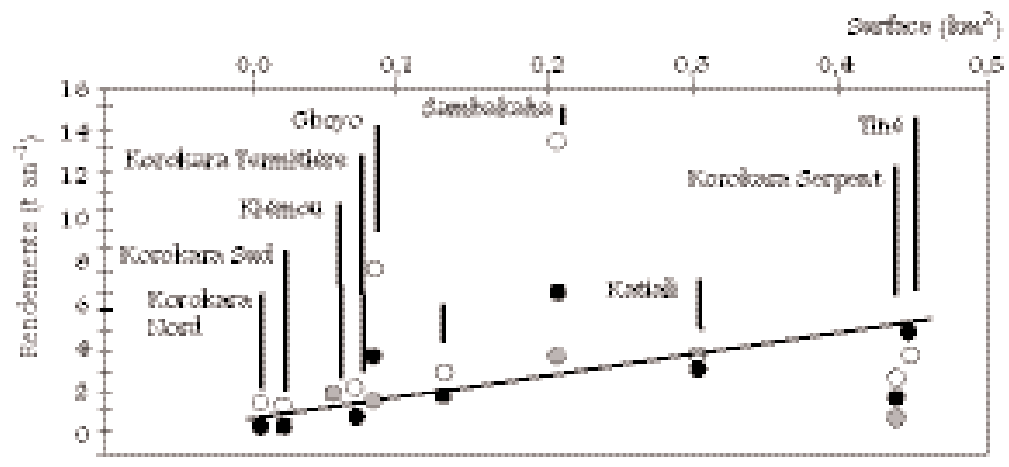

FIG. 2 - Rendements ( $t$ an $\left.^{-1}\right)$ de la pêche artisanale en fonction de la surface maximale du lac $\left(\mathrm{km}^{2}\right)$, dans 10 petites retenues du nord de la Côte d'Ivoire (noir : données de 1996-1998; gris : données de 1994 (DA Costa et al., 1998) ; blanc: rendements prédits, sous l'hypothèse d'une standardisation de l'effort de pêche à 30 jours pêcheur-1 mois $\left.^{-1}\right)$. La droite de régression $\left(R^{2}=0,57\right)$ est celle donnée par CRUL (1992 b) sur 15 lacs et réservoirs africains de petite taille (surface $<10 \mathrm{~km}^{2}$ ); captures $=9,88 \times$ surface ${ }^{0,79}$. 
Tabl. II - Rang des premières espèces les plus importantes (en poids) dans les pêches artisanales (Art.) et expérimentales (Exp.).

\begin{tabular}{|l|c|c|}
\hline Espèces & Art. & Exp. \\
\hline Oreochromis niloticus & 1 & 5 \\
Sarotherodon galilaeus & 2 & 3 \\
Clarias anguilllaris & 3 & 2 \\
Tilapia zillii & 4 & 1 \\
Heterotis niloticus & 5 & 8 \\
Schilbe intermedius & 14 & 4 \\
\hline
\end{tabular}

Tabl. III - Chiffres d'affaires par retenue et par an, estimés sur la base de l'effort

de pêche observé (tabl. I), et d'un prix de vente moyen, minimum et maximum respectivement de 375,250 et 500 F CFA kg-1. $^{-1}$.

\begin{tabular}{|l|r|r|r|}
\hline & \multicolumn{2}{|c|}{ Chiffres d'affaires estimés (F CFA) } \\
\hline \multirow{2}{*}{ Korokara Sud } & 12750 & minimum & maximum \\
Korokara Nord & 20250 & 8500 & 17000 \\
Korokara Termitière & 100875 & 13500 & 27000 \\
Nambengué & 575625 & 67250 & 134500 \\
Korokara Serpent & 615750 & 383750 & 767500 \\
Katiali & 1198875 & 410500 & 821000 \\
Gboyo & 1358250 & 799250 & 1598500 \\
Tiné & 1838625 & 1225750 & 2451500 \\
Sambakaha & 2559000 & 1706000 & 3412000 \\
\hline
\end{tabular}

Les chiffres d'affaires moyens estimés sur la base de l'effort de pêche annuel recensé et d'un prix de vente moyen de $375 \mathrm{FCFA} \mathrm{kg}^{-1}$ sont extrêmement variables (tabl. III).

Les recettes demeurent négligeables au niveau des plus petites retenues, comme à Korokara Sud et Nord, mais deviennent substantielles pour les réservoirs les plus grands et (ou) les plus intensément exploités (comme à Tiné et Sambakaha, respectivement).

Le chiffre d'affaire moyen par retenue est de l'ordre de 920000 F CFA an-1, ce qui représente, rapporté aux 219 réservoirs recensés lors de l'inventaire des retenues du nord du pays (LE GUEN et Tito dE M oraIs, 2001), un montant de l'ordre de 200 millions F CFA.

Les estimations des surfaces en eau par télédétection (Gourdin et al., ce volume) ont montré que dans cette région, il convenait de distinguer deux classes de réservoirs de tailles différentes ( $85 \%$ des réservoirs ont 
en moyenne une surface en eau de 4,5 ha et $15 \%$ une surface moyenne de 35,5 ha). Pour l'ensemble des 219 réservoirs de la région, la surface en eau est ainsi estimée à $20 \mathrm{~km}^{2}$ et le chiffre d'affaire moyen par unité de surface exploitée est alors voisin de 10 millions de F CFA km².

Même si cette estimation masque la très forte hétérogénéité des situations, tant en terme de productivité des écosystèmes que d'intensité d'exploitation, elle rend compte du poids économique de cette filière à l'échelle régionale.

La production moyenne annuellement exploitée est de l'ordre de 2,25 t par retenue, soit environ $492 \mathrm{t} \mathrm{an}^{-1}$ pour l'ensemble des 219 retenues de la région.

Ces chiffres modestes sont à considérer à l'aune de la dispersion des aménagements dans les zones rurales nord-ivoiriennes.

Dans un cas comme dans l'autre, ces chiffres rendent toutefois compte, et de façon vraisemblablement minorée, des échanges en monétaire mais aussi en protéines associés à l'activité halieutique.

Discussion

La mesure de l'effort de pêche retenue (kg pêcheur-1 jour-1) est critiquable car les engins utilisés diffèrent d'un pêcheur à l'autre, de même que la durée effective de la pêche, ce qui conditionne l'efficacité et le résultat des pêches. Cependant, cette mesure étant la plus souvent et la plus aisément collectée, c'est elle qui est utilisée dans la très grande majorité des études sur les pêches artisanales des eaux intérieures africaines (CRUL, 1992 b). C'est le nombre de pêcheurs par plan d'eau qui est indiqué comme valeur de l'effort de pêche dans les grands recueils et bases de données sur l'Afrique (Crul, 1992 a ; Vanden Bosche et BernacseK, 1990 a, 1990 b, 1991).

Dans le cas des petites retenues étudiées ici, les types d'engins et leurs modalités d'utilisation apparaissent suffisamment proches pour que nous puissions considérer cette unité comme pertinente. Bien que le modèle de Crul ne soit fondé que sur des réservoirs de surfaces supérieures à celles des retenues étudiées ici, il s'agit du modèle le plus proche que nous ayons trouvé (surface $<10 \mathrm{~km}^{2}$ ). Ce modèle linéaire, avec toutes les limitations liées à l'incertitude sur les quantités débarquées et sur l'effort de pêche est, à notre connaissance, le meilleur outil disponible pour associer surfaces et rendements dans les très petites retenues africaines. 
Il apparaît que, dans l'état actuel, le niveau d'exploitation des petits barrages se situe dans l'ordre de grandeur de ce qui est observé ailleurs en Afrique subsaharienne. Eu égard à leur faible surface $\left(<1 \mathrm{~km}^{2}\right)$, le taux de pêcheurs par $\mathrm{km}^{2}$ est évidemment excessif par rapport aux normes admises par la FAO (2 pêcheurs $\mathrm{km}^{-2}$, Henderson et Welcomme, 1974). C'est la faible efficacité des engins employés, associée souvent à un faible nombre de jours de pêche par pêcheur par mois, qui permet actuellement aux niveaux d'exploitation de ne pas atteindre des valeurs trop élevées.

Si, dans la plupart des cas, une augmentation du nombre de jours de pêche par pêcheur et par mois semble réalisable sans atteindre des niveaux de surexploitation, chaque cas doit cependant être étudié de façon spécifique. Certaines retenues, telles que Sambakaha par exemple, présenteraient en effet un niveau d'exploitation très élevé en cas d'augmentation de la durée de la pêche, a fortiori si la qualité et l'efficacité des engins étaient améliorées simultanément. En tout état de cause, toute intervention tendant soit à améliorer l'efficacité des engins, soit à augmenter la durée effective de la pêche, doit être faite avec prudence.

La comparaison des compositions des pêches expérimentales et des pêches artisanales montre qu'il n'existe apparemment pas d'espèces abondantes qui échapperaient aux captures des pêcheurs. En revanche, il a été montré qu'au sein de ces écosystèmes (Da Costa et al., ce volume), des niches trophiques paraissent vacantes, entraînant une sous-exploitation des biomasses primaires produites et accumulées dans les retenues par les communautés de poissons actuellement en présence. L'introduction de taxons spécialisés (Labeo sp. en l'occurrence) pourrait permettre alors d'améliorer l'efficacité des transferts énergétiques au sein des barrages vers des espèces exploitables, d'élargir le spectre des peuplements exploités, et donc d'en augmenter la valeur.

Parmi les retenues s'écartant le plus du modèle (fig. 2), deux d'entre elles, Gboyo et Sambakaha, présentent des rendements particulièrement élevés sans révéler pour autant de signe de surexploitation. Plusieurs hypothèses peuvent l'expliquer :

- les pêcheurs de ces retenues feraient preuve d'une efficacité supérieure à la moyenne. Ces retenues sont exploitées par des pêcheurs " attitrés», I'un d'eux notamment, à Sambakaha, constituant une remarquable exception à la situation prévalente partout ailleurs dans la région, puisque ce pêcheur est un autochtone sénoufo, formé à la pêche sur le barrage de Kossou lorsque les projets de développement de la pêche sur ce grand barrage avaient été mis en œuvre. II est de plus 


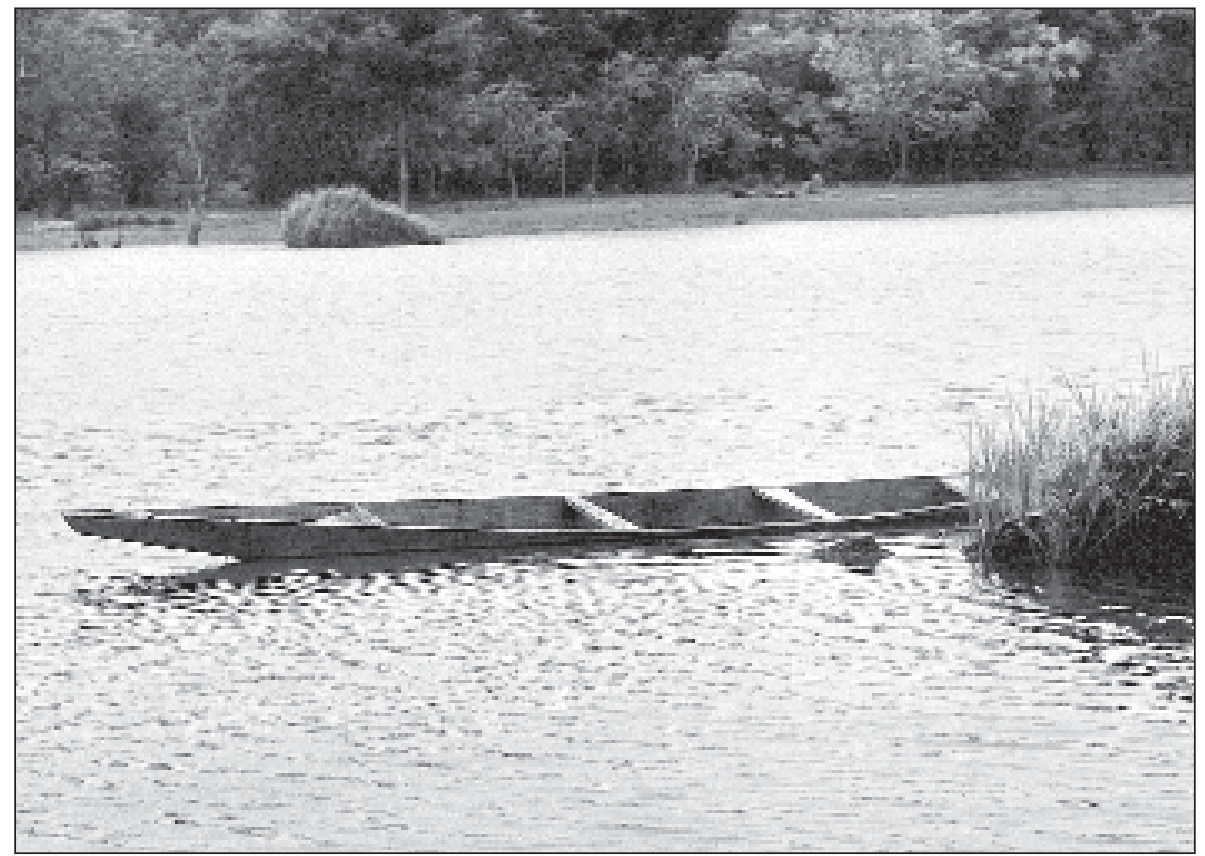

Pirogue neuve à Sambakaha. Une telle embarcation correspond à un investissement véritable : plusieurs centaines de milliers de francs CFA sont en effet nécessaires pour en financer la construction. Les encadreurs de la Sodepra avaient initialement imaginé que les barques et engins de pêche seraient directement fournis aux pêcheurs par les Comités de gestion. Cette consigne a disparu avec le temps.

remarquablement outillé et possède en particulier un jeu de sennes qu'il utilise parfois à Sambakaha mais qu'il lui arrive aussi de louer à d'autres comités de gestion de la zone souhaitant exploiter leurs propres barrages. Le comité de gestion de Sambakaha est par ailleurs I'un des plus actifs de la région ;

- les engins de pêche utilisés sont de meilleure qualité, plus nombreux ou plus grands que ceux utilisés par les autres pêcheurs - voir précédemment ;

- les deux retenues sont particulièrement productives. Elles font en effet partie des retenues où les pêches expérimentales ont été les plus performantes, supérieures en poids à ce qui a par exemple été observé à Katiali, où pourtant la surface de la retenue est 1,5 à 3 fois plus grande.

À l'inverse, la retenue de Korokara Serpent apparaît très peu productive d'après nos enquêtes. Dans ce village, de nombreux conflits émaillent les relations entre le comité de gestion et les usagers et exploitants des plans d'eau. La pêche y est régulièrement interrompue en raison de litiges relatifs au montant de la rente d'exploitation que les pêcheurs ont à payer. Les risques de sous-évaluation de l'exploitation et de la productivité du réservoir, en raison d'une sous-estimation des débarquements, y sont réels. 
Si toutefois ces différences de productivité de pêche se confirmaient, il faudrait en rechercher le déterminisme dans les conditions édaphiques et d'habitat spécifiques aux différents sites. Les caractéristiques intrinsèques des bassins versants, les modalités de leur mise en valeur et la diversité et l'intensité des usages dont les sites sont eux-mêmes l'objet peuvent également être des facteurs discriminants.

L'exploitation halieutique des retenues pastorales fournit annuellement a minima une production de près de $500 \mathrm{t}$ de poissons par an. Cette production est de l'ordre de grandeur de celles de plusieurs grands barrages du pays (tabl. IV). Les quantités débarquées des petits barrages du nord de la Côte d'Ivoire font l'objet d'une commercialisation directe, le plus souvent locale, et correspondent à un apport significatif en protéines animales, notamment en zone rurale. La dispersion des aménagements autorise en effet une distribution ponctuelle, mais large, de ces produits frais qui peuvent constituer un complément nutritionnel significatif. Cet aspect, toujours évoqué par les agences de développement, n'a jamais été directement abordé et mériterait d'être précisément quantifié.

\begin{tabular}{|c|c|c|c|}
\hline & Barrages & Surface $\left(\mathrm{km}^{2}\right)$ & Production ( $\tan ^{-1}$ ) \\
\hline & Petits Barrages & 20 & 492 \\
\hline & Faé & 50 & 180 \\
\hline & Taabo & 60 & 650 \\
\hline & Ayamé & 120 & 960 \\
\hline & Buyo & 650 & 5000 \\
\hline $\begin{array}{r}\text { Tabl. IV - Production halieutique des grands } \\
\text { (source : MINAGRA, 1993) } \\
\text { et petits barrages de Côte d'Ivoire. }\end{array}$ & Kossou & 750 & 8000 \\
\hline
\end{tabular}

Les recettes annuelles issues de l'exploitation halieutique (a minima 200 millions de F CFA) sont pour partie redistribuées sous forme de rente vers les comités de gestion. Cette pratique est héritée des consignes laissées par la Sodepra, ce qui explique encore aujourd'hui I'intérêt que ces institutions villageoises accordent à l'activité halieutique. Cette redistribution permet également de comprendre les jeux de pouvoir qui se développent à l'intérieur des communautés villageoises pour le contrôle des comités de gestion (Coulibaly et al., ce volume). Elle permet aussi vraisemblablement de profiler des leviers d'action dans la perspective d'une intensification de l'exploitation et de son efficacité. 
En se substituant aux autorités des Eaux et Forêts qui à l'échelle nationale distribuent les droits de pêche et en réglementent la pratique, les comités instituent de facto mais implicitement un contexte où l'appropriation de la ressource et des revenus tirés de son exploitation demeure ambiguë.

L'exploration et l'exploitation «pionnières» des petits barrages correspondent en ce sens à une stratégie efficace de la part des pêcheurs, marquée par l'itinérance et le court terme. Intensifier l'activité de quelque manière que ce soit pour accroître les recettes ne paraît pas pertinent pour les pêcheurs dans le contexte actuel, tant la rente grève leurs revenus. À l'inverse, pour les comités, intervenir sur la productivité halieutique des barrages ne se justifie pas puisque, pour eux, l'exploitation étant réalisée par d'autres, les bénéfices immédiats de l'amélioration de l'exploitation ne leur profiteraient pas. Koffi (1992) évoquait le «marché de dupe» qui lie comités et exploitants. Le terme reste d'actualité, et le restera tant que les droits de propriétés et d'usages ne seront pas clarifiés.

\section{Annexe}

\section{Réservoirs étudiés}

Les codes indiqués correspondent à ceux utilisés sur la figure 1 .

Tous les sites de Korokara sont représentés sur cette carte avec le même code $(\mathrm{Kr})$. Les coordonnées et les surfaces indiquées ont été collectées dans le cadre du programme Petits Barrages.

\begin{tabular}{|l|c|c|c|c|}
\hline Site & Code & Latitude N & Longitude W & Surface $\left(\mathbf{k m}^{2} \mathbf{)}\right.$ \\
\hline Korokara Nord & $(\mathrm{Kr})$ & $09^{\circ} 53^{\prime} 29^{\prime \prime}$ & $05^{\circ} 37^{\prime} 36^{\prime \prime}$ & 0,01 \\
Korokara Sud & $(\mathrm{Kr})$ & $09^{\circ} 52^{\prime} 40^{\prime \prime}$ & $05^{\circ} 38^{\prime} 07^{\prime \prime}$ & 0,02 \\
Kiémou & $(\mathrm{Km})$ & $09^{\circ} 02^{\prime} 20^{\prime \prime}$ & $05^{\circ} 34^{\prime} 08^{\prime \prime}$ & 0,06 \\
Korokara Termitière & $(\mathrm{Kr})$ & $09^{\circ} 54^{\prime} 47^{\prime \prime}$ & $05^{\circ} 36^{\prime} 40^{\prime \prime}$ & 0,08 \\
Gboyo & $(\mathrm{Gb})$ & $09^{\circ} 26^{\prime} 32^{\prime \prime}$ & $05^{\circ} 07^{\prime} 55^{\prime \prime}$ & 0,09 \\
Nambengué & $(\mathrm{Nb})$ & $10^{\circ} 03^{\prime} 12^{\prime \prime}$ & $05^{\circ} 18^{\prime} 57^{\prime \prime}$ & 0,13 \\
Sambakaha & $(\mathrm{Sb})$ & $09^{\circ} 24^{\prime} 09^{\prime \prime}$ & $05^{\circ} 06^{\prime} 21^{\prime \prime}$ & 0,21 \\
Katiali & $(\mathrm{Ki})$ & $09^{\circ} 48^{\prime} 45^{\prime \prime}$ & $05^{\circ} 57^{\prime} 09^{\prime \prime}$ & 0,31 \\
Korokara Serpent & $(\mathrm{Kr})$ & $09^{\circ} 51^{\prime} 46^{\prime \prime}$ & $05^{\circ} 36^{\prime} 11^{\prime \prime}$ & 0,44 \\
Tiné & $(\mathrm{Tn})$ & $09^{\circ} 31^{\prime} 17^{\prime \prime}$ & $05^{\circ} 32^{\prime} 04^{\prime \prime}$ & 0,45 \\
\hline
\end{tabular}




\section{Références}

Baijot E., Moreau J., Bouda S., 1994 Aspects hydrobiologiques et piscicoles des retenues d'eau en zone soudanosahélienne. Centre technique de Coopération agricole et rurale (CTA), Commission des communautés européennes (CCE), Bruxelles, 250 p.

Corbin D., Étienne J., 1998 - Cartographie de 8 retenues de Côte d'Ivoire étudiées dans le cadre du programme « Petits Barrages ». IRD Bouaké, rapport multigr., $89 \mathrm{p}$.

CRUL R. C. M., 1992 a - Database on the inland fisheries resources of A frica (DIFRA). Description d'une base de données portant sur les ressources halieutiques des eaux intérieures de I'Afrique. CIFA Occas. Pap., 17, 21 p.

CRul R. C. M., 1992 b - Modèles pour l'estimation des rendements potentiels en poisson des eaux intérieures africaines. CIFA Occas. Pap., $16,23 \mathrm{p}$.

Da Costa K. S., Traoré K., Tito de MORAIS L., 1998 - Effort de pêche et production exploitée dans les petites retenues du Nord de la Côte d'Ivoire. Bull. Fr. Pêche Piscic., 348 : 65-78.
Duponchelle F., Cecchi P., Corbin D., Nunez J., Legendre M., 2000 Variations in fecundity and egg size of female Nile tilapia, Oreochromis niloticus, from man-made lakes of Côte d'Ivoire. Environ. Biol. Fish., 57 : 155-170.

Henderson H. F., Welcomme R. L., 1974 - The relationship of yield to morpho-edaphic index and number of fishermen in African inland fisheries. CIFA Occas. Pap., 1, 19 p.

IDESSA, 1992 - Valorisation du potentiel piscicole des barrages hydro-agro-pastoraux du nord de la Côte d'Ivoire. Rapport préliminaire. Projet «Petits Barrages». Cl/CRDI, CNRA Bouaké, Côte d'Ivoire, $181 \mathrm{p}$.

Koffl C., 1992 - «Aspects socioéconomiques des pêches et de la commercialisation des ressources des plans d'eau hydro-agropastoraux du nord de la Côte d'Ivoire». In IDESSA (éd.) : Valorisation du potentiel piscicole des barrages hydro-agro-pastoraux du nord de la Côte d'Ivoire, CNRA, Bouaké, Côte d'Ivoire : 143-163 + annexes.
Le Guen T., Tito de Morais L., 2001 Diversité des utilisations agricoles associées aux retenues d'eau du nord de la Côte d'Ivoire. Cahiers d'Outre- Mer, 54 (215) : 283-304.

Minagra, 1993 - Plan directeur du développement agricole 1992-2015. A bidjan, Côte d'Ivoire, 166 p.

Moreau J., De Silva S. S., 1991 Predictive fish yield models for lakes and reservoirs of the Philippines, Sri Lanka and Thailand. FAO Fish. Tech. Rep., 319, 42 p.

Vanden Bosche J. P., Bernacsek G., 1990 a - Source book on the inland fishery resources of Africa. Vol I. CIFA Tech. Pap., 18 (1), 411 p.

Vanden Bosche J. P., Bernacsek G., $1990 \mathrm{~b}$ - Source book on the inland fishery resources of Africa. Vol II. CIFA Tech. Pap., 18 (2), 240 p.

Vanden Bosche J. P., Bernacsek G., 1991 - Source book on the inland fishery resources of Africa. Vol III. CIFA Tech. Pap., 18 (3), 219 p.

Yté W. A., Kouassi N. C., Ayekoue P., Soro S., 1997 - Peuplement zooplanctonique de quelques retenues agro-pastorales du Nord de la Côte d'Ivoire : les rotifères. Agron. Afr., 9 (2) : 125-134. 